\title{
Mechanical Properties of Mitral Allografts Are Not Reasonably Influenced by Cryopreservation in Sheep Model
}

\author{
J. HLUBOCKÝ ${ }^{1}$, A. MOKRÁČEK ${ }^{2}$, V. NOVÁČEK ${ }^{3}$, J. VOJÁČEK ${ }^{4}$, J. BURKERT $^{3}$, \\ P. KOCHOVÁ ${ }^{5}$, J. KLEPÁČEK $^{6}$, J. PEPPER $^{7}$, J. S̆PATENKA $^{3}$
}

${ }^{1}$ Department of Cardiovascular Surgery, General University Hospital, Prague, Czech Republic, ${ }^{2}$ Department of Cardiac Surgery, Hospital České Budějovice, Czech Republic, ${ }^{3}$ Department of Cardiac Surgery \& Transplant Center, University Hospital Motol, Prague, Czech Republic, ${ }^{4}$ Department of Cardiac Surgery, University Hospital, Hradec Králové, Czech Republic, ${ }^{5}$ Department of Mechanics, University of West Bohemia, Plzeň, Czech Republic, ${ }^{6} \mathrm{New}$ Technologies Centre, University of West Bohemia, Plzeň, Czech Republic, ${ }^{7}$ Academic Department of Cardiac Surgery, Royal Brompton Hospital, London, UK

Received September 3, 2010

Accepted December 22, 2010

On-line March 14, 2011

\begin{abstract}
Summary
A mitral allograft is used exceptionally in the mitral, as well as in the tricuspid position, mostly as an experimental surgical procedure. The authors decided to evaluate the possibility of inserting a cryopreserved mitral allograft into the tricuspid position in a sheep experimental model. Within the framework of this experimental project the mechanical properties of the cryopreserved mitral allograft were tested. A novel methodology studying the functional unit composed of mitral annulus, leaflet, chordae tendinaea, and papillary muscle is presented. A fiveparameter Maxwell model was applied to characterize the viscoelastic behavior of sheep mitral valves. A control group of 39 fresh mitral specimens and a test group of 13 cryopreserved mitral allografts from tissue bank were tested. The testing protocol consisted of six loading cycles with $1 \mathrm{~mm}$ elongation every $5 \mathrm{~min}$. There was no significant difference in the mean values of the determined parameters $(p>0.05)$ which confirms the main hypothesis that cryopreservation does not influence significantly material parameters characterizing the tissue mechanics. Slight discrepancy is observed in variances of viscous parameters suggesting that the values of the test group may be spread over larger interval due to the treatment.
\end{abstract}

\section{Key words}

Mitral allograft • Cryopreservation • Maxwell model • Viscoelastic properties • Mitral valve

\author{
Corresponding author \\ Vít Nováček, Transplant Center University Hospital Motol, \\ V Uvalu 84, 15006 Prague 5, Czech Republic. E-mail: \\ vit.novacek@gmail.com
}

\section{Introduction}

During last half century the heart valve surgery has been developed - recently, heart valves are repaired, if possible, or replaced, mainly using commercially available prostheses. Both lines of valvular substitutes, mechanical as well as biological ones, underwent huge technological development. Mechanical prostheses remain popular for their durability, while the biological (xenograft tissue) valves are preferred for low thrombogenicity. Both heart valve prosthesis types are simple to implant, but the main limitation remains the need for lifelong anticoagulation therapy in the mechanical prosthesis group and structural valve deterioration after 10 to 15 years in the xenograft group, respectively.

Although first experiments with mitral allograft (MA) have been reported even earlier than those with aortic allografts, MA was never widely used in clinical practice (Bodnar 2004, Hubka et al. 1966). The durability of MA was very disappointing even when operated by very experienced surgeons. Many disadvantages of MA 
in mitral position disappear when it is implanted into tricuspid position, e.g. to low pressure system. Patients with tricuspid valve bacterial endocarditis, in particular, can theoretically benefit from MA transplantation, and that awakened our interest.

We decided to evaluate methods of tricuspid valve replacement by mitral allograft in a sheep model. The allograft heart valve tissue banking facilities are essential for starting the MA transplantation program. For aortic and pulmonary allografts banking many protocols of processing and cryopreservation are available, but no recent protocol was published for MA banking. As a basic step we decided to use the same protocol, which our bank uses for human aortic and pulmonary allografts in our sheep experiments. At experimental settings the simple tearing test performed by the surgeon proved quite reliable for determining mechanical tissue properties. Short term as well as long-term sheep experimental results proved to be promising (Vojáček et al. 2006).

In aortic allografts no detectable differences were found between the mechanical behavior of the cryopreserved allograft aortic leaflets and fresh tissue (Vesely et al. 1990). There is not much data concerning mechanical properties of MA.

\section{Materials and Methods}

A control group of 39 fresh sheep MA and a test group of 13 cryopreserved sheep MA were studied. Test group was economically more demanding while higher number of fresh allografts was available so that we did measurements on all of them.

The study was performed according to guidelines \& practice, established by institutional Committee for Animal Care and Use, and was in compliance with the Czech Republic legislation. All animals received human care in compliance with the European Convention on Animal Care and the study was approved by the Institutional Ethical Committee.

Surgical exposure for the MA harvesting was achieved via a right anterolateral thoracotomy, through the 5 th intercostal space. Afterwards the animals were put to death by intravenous administration of thiopentone (Thiopental, ICN, Czech Republic a.s. - $10 \mathrm{mg} / \mathrm{kg}$ ) and potassium chloride (Kalium chloratum, Zentiva a.s., Praha $-20 \mathrm{ml} / \mathrm{kg}$ ). Their hearts were explanted under sterile conditions, mitral valves were harvested with rims of left atrial and left ventricular muscle and with the entire subvalvular apparatus, including both papillary muscles.

After harvesting, 39 fresh MAVs were stored in saline, in cool box at +5 to $+7{ }^{\circ} \mathrm{C}$, and were mechanically tested within 24 hours. Another 13 MAVs were processed according to the standard protocol of the Cardiovascular Tissue Bank, Transplant Center, University Hospital Motol, Prague (Špatenka 1997). They were deposited directly into the cultivation medium E 199 with the antibiotic cocktail - cefuroxime $0.2 \mathrm{mg} / \mathrm{ml}$, (Zinacef, GlaxoWellcome) + piperacillin $0.2 \mathrm{mg} / \mathrm{ml}$ (Pipril, Lederle) + amikacin $0.1 \mathrm{mg} / \mathrm{ml}$ (Amikin, Bristol-Myers Squibb) + fluconazol $0.1 \mathrm{mg} / \mathrm{ml}$ (Diflucan, Pfizer). After $24 \mathrm{~h}$ storage at the temperature of $37.0{ }^{\circ} \mathrm{C}$ the valves were kept at +5 to $+7{ }^{\circ} \mathrm{C}$ over the period of 3-5 days. Then they were transferred under sterile conditions into the cryoprotective solution (E 199 with $10 \%$ dimethylsulfoxide) in a laminar flow box, and sealed into plastic bags (Gambro Hemofreeze/Haemo bags NPBI BV DF 1200, the Netherlands) using twolayer technique. Finally, MA were programmed cooled (between temperatures of +10 to $-60{ }^{\circ} \mathrm{C}$ at the rate of $-1{ }^{\circ} \mathrm{C} / \mathrm{min}$ ) and then stored in liquid phase of liquid nitrogen $\left(-196^{\circ} \mathrm{C}\right)$ in a separate container. Average storage time in tissue bank was 5.3 months (from 3.5 to 12 months). During the transportation from cryostore to the experimental facilities MA were kept in a container CP500 (Wharton \& Taylor, USA) at gaseous phase of liquid nitrogen (temperature below $-140{ }^{\circ} \mathrm{C}$ ). Thirty minutes before the experiment they were removed from the container and thawed in a standard way used for human aortic and pulmonary allografts $(15 \mathrm{~min}$ in room temperature followed by 15 mins in $37{ }^{\circ} \mathrm{C}$ water bath). We used exactly the same procedure for human tissue in clinical practice, as for the experimental MA transplantation in the sheep model.

In the experimental laboratory we used a similar shape of sample in all cases so we were measuring the mechanical properties of all parts of mitral valve at one time. Samples always contained the mitral annulus, part of the anterior leaflet, corresponding chordae tendineae and the postero-medial papillary muscle. For mechanical testing, the MA tissue was fixed to the traction machine jaws with mitral annulus and papillary muscle.

Stepwise stress-relaxation measurements were made on all tissue samples. Zwick Roell Z050 (Zwick GmbH \& Co, Germany, http://www.zwick.de) traction machine equipped with pneumatic grips and $200 \mathrm{~N}$ loading cell was used for the MA tissue testing. 


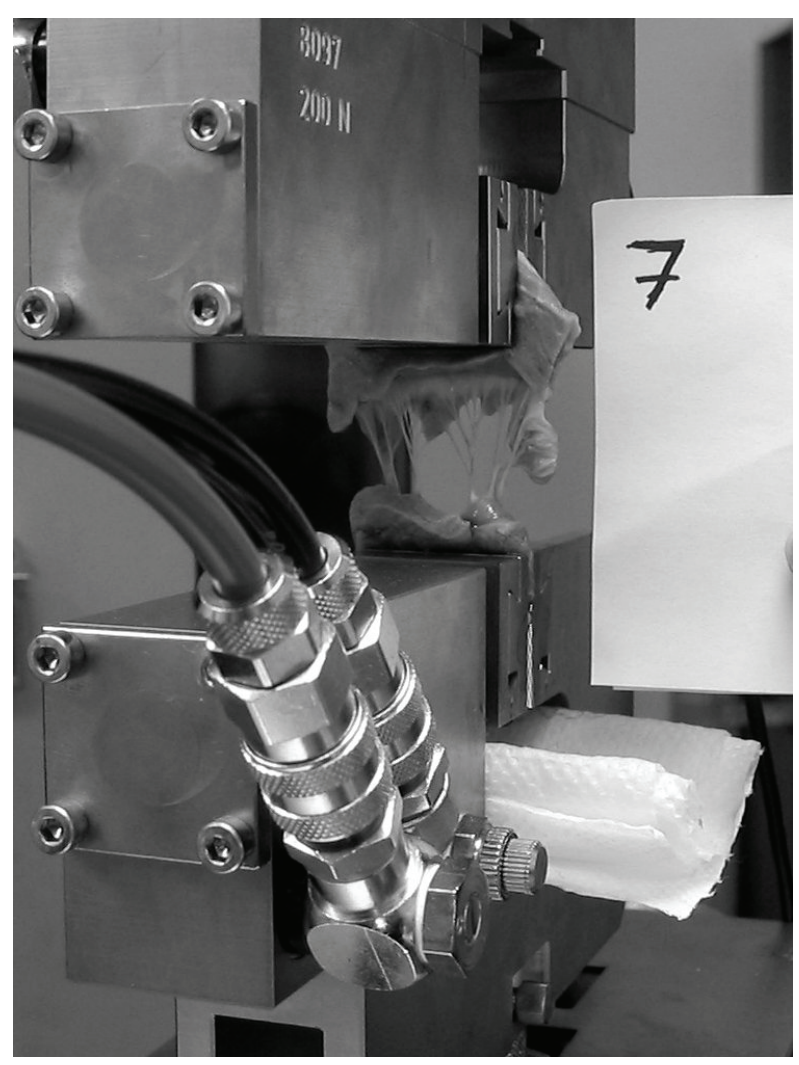

Fig. 1. Heart valve tissue sample between the pneumatic grips of the $200 \mathrm{~N}$ loading cell of the experimental apparatus Zwick Roell Z050.

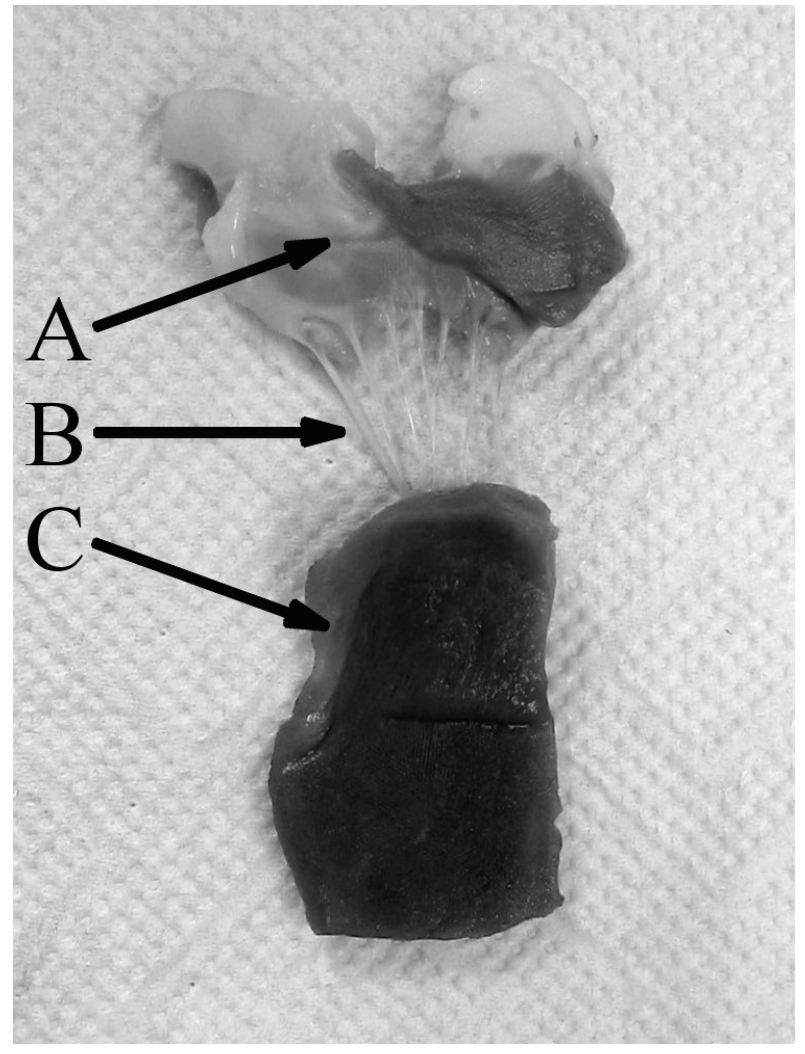

Fig. 2. Valve tissue sample after the experiment. Part of anterior leaflet of mitral valve $(A)$ with its chordae tendineae $(B)$ and part of papillary muscle $(C)$.
The specimens were fixed between the grips of the apparatus with a free length corresponding to the specimens' dimensions (Fig. 1). The samples (Fig. 2) were stretched in steps of 1 millimeter every $5 \mathrm{~min}$ and the loading protocol consisted in six loading cycles. The time elapsed from the beginning of the step was chosen to reach approximately the steady state. The forceelongation curves were recorded.

The five element generalized Maxwell model was used in this study for the description of the relaxation behavior of the tissue. A simple Maxwell body includes a viscous element, $\eta$, and an elastic element, $E$, connected in series. Generalized Maxwell models consist of some simple Maxwell bodies coupled in parallel. In addition, an elastic element, $E_{P}$, may be connected in parallel to them. In stress relaxation experiments, the applied stretch $e(t)=\hat{e}$ is instantaneous and constant throughout the loading cycle. Constitutive equation of the generalized Maxwell model consisting in $n$ Maxwell bodies with parallel elastic element $E_{P}$ may be written as:

$F(t)=\hat{e} E_{P}+\sum_{i=1}^{n} \hat{e} E_{i} \exp \left(-t / \tau_{i}\right)$

where the time constant $\tau_{i}=\eta_{i} / E_{i}$ represents the corresponding relaxation time for one step of stretching and $\eta_{i}$ and $E_{i}$ denote the viscous and elastic modulus of $i$-th Maxwell body, respectively. For five element Maxwell model with $n=2$, the above equation is the final force-time relation with five material parameters $E_{P}$, $E_{1}, E_{2}, \eta_{1}$, and $\eta_{2}$ to be identified.

\section{Results}

The above equations describe a single step of stretching at constant values of the parameters $E_{P}, E_{1}, E_{2}$, $\eta_{1}$, and $\eta_{2}$. Their values are different for each relaxation step. To identify these parameters, a direct exponential fitting to the experimental data was performed using statistical software $\mathrm{R}$ (The $R$ Project for Statistical Computing, http://www.R-project.org). The fitting process was the Gauss-Newton algorithm based on the nonlinear least-squares method. A set of material parameters was determined for each stretching step and for each specimen of the control group of fresh tissue and the test group of thawed cryopreserved MA, respectively. Coefficient of correlation $R$ of the fitting procedure was better than 0.99 for all specimens and every loading cycle. 
From the total number of 52 heart valves, the control group of fresh tissue contained 39 specimens while the test group of cryopreserved MA was represented by 13 specimens. The fitted values of material parameters were examined cycle by cycle. For each loading cycle, the outliers were carefully detected using standard techniques of exploratory data analysis and they were removed from the statistical files. Table 1 summarizes the mean values and standard deviations of determined viscoelastic parameters for both the control and test group as well as the number of outliers.

For each viscoelastic parameter and each loading cycle, null hypotheses were formulated stating that the means and the variances of the two groups under consideration do not differ significantly. To test the hypotheses, t-test and F-test were carried out in $\mathrm{R}$ statistical package. The assumed normality of the populations was tested and confirmed before the execution of the above mentioned tests. Both statistical tests were carried out at $95 \%$ confidence level.

The mean values of identified elastic moduli $E_{P}, E_{1}$, and $E_{2}$ do not manifest any pronounced dominancy of one group of specimens over the other. Order of magnitude of all three elastic moduli ranges from $10^{2}$ to $10^{3} \mathrm{~N} / \mathrm{m}$.

The identified values of the parallel elastic modulus $E_{P}$ range from $0.44 \pm 0.24 \mathrm{kN} / \mathrm{m}$ in the first loading cycle to $4.65 \pm 1.70 \mathrm{kN} / \mathrm{m}$ in the last loading cycle for the control group and from $0.58 \pm 0.19 \mathrm{kN} / \mathrm{m}$ to $3.91 \pm 1.79 \mathrm{kN} / \mathrm{m}$ for the test group. In the first loading cycle, the modulus of the test group is slightly higher than that of the control group while its standard deviation remains lower. Control group modulus is then slightly higher in the next loading cycles.

Serial elastic modulus $E_{l}$ is lower than parallel elastic modulus $E_{P}$ but of the same order. For the control group, the values range from $0.29 \pm 0.13 \mathrm{kN} / \mathrm{m}$ to $1.40 \pm 0.43 \mathrm{kN} / \mathrm{m}$, and for the test group from $0.36 \pm 0.12 \mathrm{kN} / \mathrm{m}$ to $1.30 \pm 0.56 \mathrm{kN} / \mathrm{m}$. The values of the test group are slightly higher than the control group values up to the fourth loading cycle, almost equal in the fifth loading cycle, and finally little bit lower in the last loading cycle.

The identified values of serial elastic modulus $E_{2}$ are very similar to those of serial elastic coefficient $E_{l}$. The identified data encompass the interval from $0.25 \pm 0.12 \mathrm{kN} / \mathrm{m}$ in the first loading cycle to $1.28 \pm 0.48 \mathrm{kN} / \mathrm{m}$ in the last loading cycle in the case of the control group and from $0.23 \pm 0.12 \mathrm{kN} / \mathrm{m}$ to
$1.18 \pm 0.64 \mathrm{kN} / \mathrm{m}$ in the case of the test group. The mean values of the test group are higher than means of the control group in the second and third loading cycle. This difference, however, is less than $0.05 \mathrm{kN} / \mathrm{m}$ up to the fourth loading cycle.

No significant difference $(p>0.05)$ was found for elastic parameters between the two groups of specimens, neither between the mean values, nor between the variances.

Test group serial viscous modulus $\eta_{l}$ has higher values than that of the control group as apparent from Table 1. Order of magnitude of viscous modulus $\eta_{1}$ lies between $10^{4}$ and $10^{5} \mathrm{Ns} / \mathrm{m}$. It is the only viscoelastic parameter determined with such strongly pronounced dominance of one specimen group. The values range from $33.75 \pm 13.36 \mathrm{kNs} / \mathrm{m}$ to $159.99 \pm 48.48 \mathrm{kNs} / \mathrm{m}$ for the control group and from $44.08 \pm 15.19 \mathrm{kNs} / \mathrm{m}$ to $163.30 \pm 90.85 \mathrm{kNs} / \mathrm{m}$ for the test group. No significant difference $(p>0.05)$ was found between the mean values of the two groups of specimens. Significant difference in variances was observed in the third, fifth, and sixth loading cycle with $p$ equal to $0.027,0.042$, and 0.004 , respectively. Test group standard deviation is more than 1.65 higher than control group standard deviation in these three critical loading cycles.

As the equality of variances is one of the assumptions of the Student's t-test, the relevance of its results in the third, fifth, and sixth loading cycle may be compromised. Nevertheless, the difference of the mean values expressed as percentage of the higher mean gives $23 \%, 25 \%, 27 \%, 14 \%, 19 \%$, and $2 \%$. It is obvious that the differences $27 \%$ in the third cycle and $19 \%$ in the fifth cycle fall into reasonable limits. Note only $2 \%$ difference in means in the sixth loading cycle. In this respect, the difference in means between the two groups remains negligible.

Viscous modulus $\eta_{2}$ of the test group has lower values than that of the control group and it has values of order of magnitude $10^{3} \mathrm{Ns} / \mathrm{m}$. Identified values of the viscous modulus $\eta_{2}$ encompass the interval from $1.63 \pm 0.79 \mathrm{kNs} / \mathrm{m}$ to $7.57 \pm 2.49 \mathrm{kNs} / \mathrm{m}$ in the control group and from $1.74 \pm 1.13 \mathrm{kNs} / \mathrm{m}$ to $5.84 \pm 3.56 \mathrm{kNs} / \mathrm{m}$ in the test group. As well as in the previous cases, no significant difference $(p>0.05)$ was found between the mean values of the two groups of specimens. Significant difference $(p=0.031)$ in variances was observed in the fourth loading cycle. However, the difference between mean values in this loading cycle is practically negligible as shown in Table 1. 
Table 1. Identified material coefficients.

\begin{tabular}{|c|c|c|c|c|c|c|c|}
\hline \multirow{2}{*}{ Parameter } & \multirow{2}{*}{ Group } & \multicolumn{6}{|c|}{ Loading cycle } \\
\hline & & 1 & 2 & 3 & 4 & 5 & 6 \\
\hline \multirow{2}{*}{$E_{P}(\mathrm{kN} / \mathrm{m})$} & control & $0.44 \pm 0.24$ & $1.09 \pm 0.78$ & $1.96 \pm 0.90$ & $2.82 \pm 1.14$ & $3.72 \pm 1.48$ & $4.65 \pm 1.70$ \\
\hline & test & $0.58 \pm 0.19$ & $1.09 \pm 0.44$ & $1.69 \pm 0.79$ & $2.41 \pm 1.08$ & $2.98 \pm 1.05$ & $3.91 \pm 1.79$ \\
\hline \multirow{2}{*}{$E_{l}(\mathrm{kN} / \mathrm{m})$} & control & $0.29 \pm 0.13$ & $0.39 \pm 0.15$ & $0.61 \pm 0.22$ & $0.85 \pm 0.28$ & $1.14 \pm 0.37$ & $1.40 \pm 0.43$ \\
\hline & test & $0.36 \pm 0.12$ & $0.48 \pm 0.13$ & $0.71 \pm 0.29$ & $0.87 \pm 0.40$ & $1.11 \pm 0.36$ & $1.30 \pm 0.56$ \\
\hline \multirow{2}{*}{$E_{2}(\mathrm{kN} / \mathrm{m})$} & control & $0.25 \pm 0.12$ & $0.29 \pm 0.12$ & $0.50 \pm 0.21$ & $0.72 \pm 0.25$ & $1.03 \pm 0.35$ & $1.28 \pm 0.48$ \\
\hline & test & $0.23 \pm 0.12$ & $0.33 \pm 0.17$ & $0.53 \pm 0.26$ & $0.69 \pm 0.36$ & $0.84 \pm 0.21$ & $1.18 \pm 0.64$ \\
\hline \multirow{2}{*}{$\eta_{I}(\mathrm{kNs} / \mathrm{m})$} & control & $33.75 \pm 13.36$ & $46.64 \pm 15.38$ & $72.29 \pm 23.84$ & $96.81 \pm 30.94$ & $130.51 \pm 44.06$ & $159.99 \pm 48.48$ \\
\hline & test & $44.08 \pm 15.19$ & $62.43 \pm 19.10$ & $99.22 \pm 39.50$ & $113.24 \pm 41.14$ & $161.62 \pm 73.61$ & $163.30 \pm 90.85$ \\
\hline \multirow{2}{*}{$\eta_{2}(\mathrm{kNs} / \mathrm{m})$} & control & $1.63 \pm 0.79$ & $2.06 \pm 0.83$ & $3.36 \pm 1.31$ & $4.46 \pm 1.63$ & $6.27 \pm 2.42$ & $7.57 \pm 2.49$ \\
\hline & test & $1.74 \pm 1.13$ & $1.94 \pm 0.86$ & $3.24 \pm 1.69$ & $4.40 \pm 2.67$ & $4.70 \pm 2.13$ & $5.84 \pm 3.56$ \\
\hline \multirow{2}{*}{ outliers } & control $(n=39)$ & 14 & 7 & 4 & 4 & 4 & 3 \\
\hline & test $(n=13)$ & 6 & 6 & 2 & 2 & 5 & 0 \\
\hline
\end{tabular}

Data are mean value \pm standard deviation and number of outliers.

\section{Discussion}

The lifelong postoperative stress on a heart valves tissue prosthesis is known to be enormous. That is why the mechanical testing of the commercial heart valve prostheses became routine during the last 50 years. Special simulators were developed for mechanical as well as for biological prostheses, and precise, scientific methods of tissue mechanics and tensile strength measurements were introduced.

As our experimental MA transplants into the tricuspid position on the sheep model were intended as a first step of clinical project we decided to examine allograft tissue quality objectively. In other words we wanted to know if our aortic \& pulmonary allograft processing and cryopreservation method was feasible for MA as well, or if we have to develop a special MA processing protocol.

For aortic and pulmonary allografts some data on mechanical properties are available. The ultrastructure and mechanics of fresh, cryopreserved, and cellular extracted porcine aortic valve leaflets were tested. Reduction in the fracture tension and increased tissue extensibility were observed. Cellular extraction preserves matrix structure and mechanics over the physiological loading range. On the contrary, the combination of extraction and fixation may lead, according to these authors, to early degenerative failure (Courtman et al. 1995).
When the mechanics of fresh, refrigerated, and frozen porcine arterial tissue was measured and statistically compared the effects and impact of common storage protocols on tissue mechanics were revealed. Subfailure stress, ultimate stress, and Young's modulus decreased significantly in refrigerated specimens while physiological, subfailure, and ultimate failure mechanics between fresh and frozen specimens were not significantly different (Stemper et al. 2007). It was reported that cryopreservation of decellularized arteries does not affect the structure and mechanical properties of the rabbit carotid artery (Fonck et al. 2008).

Exhaustive overview of the biomechanics of heart valves and their function including solid and fluid mechanics and fluid-structure interaction studies have been published recently (Sacks et al. 2009). This paper shows that most of the research concerned with heart valve mechanics has been conducted on aortic valves. Indeed, only a few studies deal with mitral valves and they are usually limited to leaflets with no interest to related heart structures. Surface strains were determined for anterior mitral valve leaflet (Sacks et al. 2002). Biaxial mechanical testing of porcine mitral valve leaflets revealed significant difference in material properties between the anterior and posterior leaflets (May-Newman and Yin 1995).

In order to quantify the influence of the cryogenic treatment on tissue mechanics, a testing 
protocol was defined and viscoelastic parameters were determined for a control group of fresh tissue specimen and a test group of cryogenically processed samples. A five-element Maxwell viscoelastic model was applied to characterize the tissue's mechanical behavior. The tissue mechanics were studied in terms of forces and elongations rather than in terms of stresses and strains since the specimens' geometrical form was very complicated and irregular that would cause the difficulties in assessing of geometrical characteristics like cross-section area.

The actual configuration of the experimental device did not allow us to immerse the specimen during the test into any liquid media, e.g. saline. Thus, the testing conditions are somewhat removed from the situation in the animal's body. In order to approach real conditions and to prevent dehydration of the specimen, the specimens were moistened during the test, the total testing time was reduced to 30 minutes and there was no preconditioning phase in the testing protocol. Preconditioning is recommended for stabilizing the internal structure of the tissue that should decrease variability and lead to interpretable results (Fung 1993). Freezing is considered as one type of preconditioning since it also may change the mechanical properties of soft tissue (Gao et al. 2010). According to the authors, results from the thawed liver tissue were generally consistent, interpretable and no other preconditioning was applied. The preconditioned state of the porcine aortic valve material is a function of the deformation history that has occurred before the preconditioning cycles and preconditioning without an adequate rest period between tests increases predictive errors (Carew et al. 2000). However, may introduce a loss of stiffness (Liao et al. 2009) or plastic deformation of tissue specimen. Anomalous decrease of stiffness of skin and myocardium tissue was attributed to the small number of cyclic loads and it was concluded that this behavior is a true phenomenon unique to load controlled deformations that results from the interplay of nonlinear effects and creep behavior (Giles et al. 2007). Test specimens are usually preconditioned by applying a cyclic load to reduce the viscoelastic effects (Ghaemi et al. 2009). However, the viscoelastic effects are of the utmost interest in our study as they introduce time-dependent phenomena like creep and/or relaxation. The aim of this study was to evaluate the overall viscoelastic behavior and to compare the influence of a specific treatment on the viscoelasticity of the tissue that is represented by a set of viscoelastic coefficients. We decided to omit the preconditioning phase as this allowed us to examine the inherent material properties of the tissue under consideration that are not modified by previously introduced deformations and/or stresses.

There are only a few studies which have focused on the influence of freezing on tissue mechanical behavior. The effect of freezing on the mechanical properties of spleen tissue has been reported to be negligible (Davies et al. 2000). No significant gross histological damage was observed in frozen liver tissue samples that were loaded within the elastic regime. Histological changes due to mechanical stresses were associated with permanent plastic deformations related to structural irregularities such as the blood vessels and bile ducts (Rabin et al. 1997). Similarly, the results of the present study do not reveal any significant difference in viscoelastic properties between fresh and frozen heart tissue.

In the present study, no significant difference between the control and testing group was found in mean values of determined parameters suggesting that the tissue processing and cryopreservation do not alter the tissue's structural components that play crucial role in its viscoelasticity. Tissue elasticity compensating mostly for the instantaneously applied load may be attributed to collagenous tissue, more precisely to the anterior leaflet and especially to the corresponding chordae tendineae. The viscous response corresponding to the relaxation of the specimen would be dominant mostly in muscular tissue. Significant differences observed in the variances of viscous parameters in certain loading cycles reflect a wider range of values of the test group compared to the control group. This difference cannot be attributed to the lack of a precondition phase since it would affect the control group as well. The difference may be due to variability in the geometry of the papillary muscle that contributes to the viscous response of the specimen and, so, to variability in its cross-section bearing the mechanical load. A similar size and geometrical shape of papillary muscle of harvested MA samples cannot be guaranteed since it depends on many factors including surgeon's judgment and experience. Moreover, attaching the muscle between the grips of the testing device may considerably change the free size of the muscle tissue that is not squeezed between the jaws and that participates on the viscous response to the applied load. Together with relatively low number of specimens in testing group and different number of specimens in each group, this factor 
may play a role in statistical analysis. Nonetheless, the results of the present study are very encouraging as they show that the tissue processing and cryopreservation do not alter significantly the overall viscoelastic behavior and mechanical performance of the tissue.

Our study shows that current allograft heart valve tissue processing and cryopreservation protocol could be applied on MA tissue as well. The mechanical properties of cryopreserved MA tissue do not differ significantly from the quality of the native mitral valve tissue in sheep model. On the basis of this experimental mechanical testing the standard allograft heart valve bank protocol will be used even for MA processing for clinical purposes.

\section{Conflict of Interest}

There is no conflict of interest.

\section{Acknowledgements}

We would like to thank the laboratories of the University of West Bohemia, namely Department of Mechanics and New Technologies Research Centre for enormous help during our experiments.

\section{References}

BODNAR E: Clinical use of homologous and heterologous mitral valves. J Heart Valve Dis 3: 468-469, 1994.

CAREW EO, BARBER JE, VESELY I: Role of preconditioning and recovery time in repeated testing of aortic valve tissues: Validation through quasilinear viscoelastic theory. Ann Biomed Eng 28: 1093-1100, 2000.

COURTMAN DW, PEREIRA CA, OMAR S, LANGDON SE, LEE JM, WILSON GJ: Development of a pericardial acellular matrix biomaterial: biochemical and mechanical effects of cell extraction, J Biomed Mater Res 28 : 655-666, 1994.

DAVIES PJ, CARTER FJ, CUSCHIERI A: Mathematical modelling for keyhold surgery imulations: a biomechanical model for spleen tissue. IMA J Appl Mathematics 67: 41-67, 2002.

FONCK E, ROY S, RÜFENACHT DA, STERGIOPULOS N: Effect of cryopreservation on the mechanical properties of decellularized arteries. J Biomech 41 (Suppl 1): 71, 2008.

FUNG YC: Bioviscoelastic solids. In: Biomechanics: Mechanical Properties of Living Tissues. Springer Verlag, New York, 1993, pp 242-314.

GAO Z, LISTER K, DESAI JP: Constitutive modeling of liver tissue: experiment and theory. Ann Biomed Eng 38: 505$516,2010$.

GHAEMI H, BEHDINAN K, SPENCE AD: In vitro technique in estimation of passive mechanical properties of bovine heart part I. Experimental techniques and data. Med Eng Phys 31: 76-82, 2009.

GILES JM, BLACK EA, BISCHOFF JE: Anomalous rate dependence of the preconditioned response of soft tissue during load controlled deformation. J Biomech 40: 777-785, 2007.

HUBKA M, SISKA K, BROZMAN M, HOLEC V: Replacement of mitral and tricuspid valves by mitral homograft. J Thorac Cardiovasc Surg 51: 195-204, 1966.

LIAO D, ZHAO J, KUNWALD P, GREGERSEN H: Tissue softening of guinea pig oesophagus tested by the tri-axial test machine. J Biomech 42: 804-810, 2009.

MAY-NEWMAN K, YIN FC: Biaxial mechanical behavior of excised porcine mitral valve leaflets. Am J Physiol Heart Circ Physiol 269: H1319-H1327, 1995.

SACKS MS, HE Z, BAIJENS L, WANANT S, SHAH P, SUGIMOTO H, YOGANATHAN AP: Surface strains in the anterior leaflet of the functioning mitral valve. Ann Biomed Eng 30: 1281-1290, 2002.

SACKS MS, MERRYMAN WD, SCHMIDT DE: On the biomechanics of heart valve function, J Biomech 42: $1804-$ $1824,2009$.

STEMPER BD, YOGANANDAN N, STINEMAN MR, GENNARELLI TA, BAISDEN JL, PINTAR FA: Mechanics of fresh, refrigerated, and frozen arterial tissue. J Surg Res 15: 236-242, 2007.

RABIN Y, OLSON P, TAYLOR MJ, STEIF PS, JULIAN TB, WOLMARK N: Gross damage accumulation in frozen rabbit liver due to mechanical stress at cryogenic temperatures. Cryobiology 34: 394-405, 1997. 
ŠPATENKA J, KOSTELKA M, KOBYLKA P, HUČÍN B, HONĚK T, LOCHMAN O, HÁJEK T, TLÁSKAL T, POVÝŠILOVÁ V, FIŠER B: Processing, cryopreservation, transportation and clinical use of allograft heart valves. (in Czech) Rozhl Chir 76: 118-125, 1997.

VESELY I, GONZALEZ-LAVIN L, GRAF D, BOUGHNER D: Mechanical testing of cryopreserved aortic allografts. Comparison with xenografts and fresh tissue. J Thorac Cardiovasc Surg 99: 119-123, 1990.

VOJÁČEK J, MOKRÁČEK A, ŠPATENKA J, VAMBERA M, ŠULDA M, ŠETINA M, BURKERT J, PAVEL P, PEPPER J: Implantation of cryopreserved mitral allograft into the tricuspid position in an experimental study in sheep: technical aspects of implantation and immediate results evaluated by epicardial echocardiography. Zentralbl Chir 131: 511-516, 2006. 